Research Paper

\title{
Cryotherapy shows no inferiority compared with radical Prostatectomy for low-risk and intermediate-risk localized Prostate Cancer: a real-world study from the SEER database
}

Kun Jin ${ }^{1 *}$, Shi Qiu ${ }^{1,2^{*}}$, Xiaonan Zheng ${ }^{1 *}$, Yanyan $\mathrm{Li}^{1}$, Shiyu Zhang ${ }^{1}$, Jiakun $\mathrm{Li}^{1}$, Xinyang Liao ${ }^{1}$, Xiang $\mathrm{Tu}^{1}$, Lu Yang ${ }^{1 \bowtie}$ and Qiang Wei ${ }^{\circledR}$

1. Department of Urology, Institute of Urology and National Clinical Research Center for Geriatrics, West China Hospital, Sichuan University, No. 37 Guoxue Xiang, Chengdu 610041, China.

2. Center of Biomedical big data, West China Hospital, Sichuan University, Chengdu, Sichuan, China.

*These authors contributed equally to this study.

$\triangle$ Corresponding author: Dr. Qiang Wei, Department of Urology, Institute of Urology, West China Hospital of Sichuan University, No. 37, Guoxue Alley, Chengdu, Sichuan, P.R. China; Post Code: 610041; E-mail: weiqiang163163@163.com; Telephone: +86 18980601425. Dr. Lu Yang, Department of Urology, Institute of Urology, West China Hospital of Sichuan University, No. 37, Guoxue Alley, Chengdu, Sichuan, P.R. China; Post Code: 610041; wycleflue@163.com, telephone: +8613541235213.

(c) The author(s). This is an open access article distributed under the terms of the Creative Commons Attribution License (https://creativecommons.org/licenses/by/4.0/). See http:/ /ivyspring.com/terms for full terms and conditions.

Received: 2019.07.10; Accepted: 2020.06.14; Published: 2020.07.29

\begin{abstract}
Background: For localized prostate cancer ( $\mathrm{PCa}$ ) with a low disease burden, whole-gland resection seems like overtreatment, while focal therapy, including cryosurgery, can achieve similar outcomes. We aimed at comparing the long-term survival outcomes of cryotherapy and radical prostatectomy (RP) and further exploring whether RP can be replaced by cryosurgery for those with low-risk PCa.

Methods: We conducted analyses from the Surveillance, Epidemiology, and End Results (SEER) database (2004-2015) and performed propensity score matching and used an instrumental variate to reduce the influence of bias and unmeasured confounders to the greatest extent.

Results: In the multivariate regression, patients who received cryotherapy had higher risk of overall mortality $(\mathrm{OM})$ (hazard ratio $[\mathrm{HR}]=2.52,95 \%$ confidence interval $[\mathrm{Cl}] 1.99-3.20, \mathrm{p}<0.001$ ), but no significant difference was observed in decreasing cancer-specific mortality (CSM) $(\mathrm{HR}=1.38,95 \% \mathrm{Cl}$ $0.63-3.03, p=0.41$ ) after adjusting the confounders. After propensity score matching, patients who underwent cryotherapy had higher OM and CSM rates $(\mathrm{HR}=2.70[95 \% \mathrm{Cl} 1.99-3.66, \mathrm{p}<0.001]$ and $\mathrm{HR}$ $=2.99$ [95\% Cl 1.19-7.48, $\mathrm{p}=0.02]$, respectively). In the IV-adjusted analyses, RP was superior to cryotherapy in decreasing OM $(\mathrm{HR}=2.52,95 \% \mathrm{Cl} 1.99-3.20)$, while no obvious decrease of CSM was observed in the comparison of RP and cryotherapy ( $\mathrm{HR}=1.38,95 \% \mathrm{Cl} 0.63-3.03)$. The subgroup analyses showed that RP displayed an obvious benefit in decreasing CSM $(H R=5.02,95 \% \mathrm{Cl} 1.30-19.39, \mathrm{p}=0.02)$ for those with a prostate-specific antigen (PSA) level higher than $10 \mathrm{ng} / \mathrm{ml}$.

Conclusion: RP ranked as the best treatment in regard to tumor control, but the advantages of cryotherapy became evident when taking functional and oncological outcomes into account, especially for low- and intermediate-risk PCa with low PSA levels.
\end{abstract}

Key words: Cryotherapy; Radical Prostatectomy; Localized; Prostate Cancer

\section{Introduction}

Prostate cancer $(\mathrm{PCa})$, the third most common malignancy in the USA, is ranked the sixth leading cause of cancer death in males, with an estimated 160,000 new cases diagnosed in 2017 [1,2]. With the 
widespread use of prostate-specific antigen (PSA) testing, an increasing number of men are diagnosed with localized PCa with a lower clinical stage, smaller volume and lower grade. Active surveillance is suggested for most individuals, due to the slight effect on overall survival. Once tumor progression occurs, radical treatments including radical prostatectomy (RP) and radiation therapy are suitable options according to EAU guidelines [3]. Although these interventions have long-term oncological control outcomes, side effects on genitourinary function cannot be avoided, such as incontinence, urinary symptoms, erectile function, and rectal side effects $[4,5]$. For those with low-grade and small-size $\mathrm{PCa}$, whole-gland resection seems like overtreatment, while focal therapy, including cryosurgery, can limit toxicity by sparing the neurovascular bundles, sphincter, and urethra [6-8].

According to one single-arm research study including 370 individuals who underwent cryosurgery, results showed that the 10-year biochemical disease-free survival rates were $80.6 \%$ and $74.2 \%$ for the low- and intermediate-risk groups [9]. However, direct comparative data and long-term oncological outcomes are still lacking, as the relevant studies focus mainly on non-comparative single-arm case series [10]. As a result, no consensus has been reached on whether cryosurgery could be an alternative therapy to cure localized PCa.

Our study aims at comparing the long-term survival outcomes between cryotherapy and radical prostatectomy and further exploring whether RP can be replaced by cryosurgery for those with low-risk PCa.

\section{Methods}

\section{Patient selection}

From the Surveillance, Epidemiology, and End Results (SEER) database, consisting of 18 cancer registries in America and accounting for $26 \%$ of the US population, we identified patients diagnosed with adenocarcinoma of the prostate (International Classification of Diseases-O-3 code: C61.9) between 2004 and 2015. The TNM stages were evaluated according to the sixth edition of the American Joint Committee on Cancer Staging Manual [11]. Inclusion and exclusion criteria are shown in detail in the flowchart (Figure 1). Patients were stratified into two treatment groups: cryotherapy and RP. These selection criteria yielded 19,554 patients in total.

\section{Propensity score matching}

To emulate randomized cohort trial design and minimize selection bias, we performed propensity score matching (1:1 ratio, with nearest-neighbor matching or caliper width of 0.05), which yielded similar patient characteristics between cryotherapy ( $\mathrm{n}$ $=2,350)$ and RP cohorts $(n=17,204)$. Adjustment variables consisted of age, biopsy Gleason score (GS), clinical tumor stage, and PSA level.

\section{Statistical analysis}

First of all, in the analysis of baseline characteristics, differences in continuous variables were evaluated with a two-tailed $t$ test and presented as the mean \pm standard deviation, whereas differences in categorical variables were compared with a two-tailed $\chi^{2}$ test (or Fisher's exact test) and presented as frequency with its proportion. Second, Cox proportional hazards regressions were performed to assess cancer-specific mortality (CSM) and overall mortality $(\mathrm{OM})$ between treatment groups in the crude models and adjusted-covariate models. In the process of matching, propensity scores were estimated with logistic regression, with treatment (cryotherapy and RP) as the outcome and age, clinical T stage, GS, and PSA level as pretreatment, prognostic covariates. The matched baseline characteristics between the two groups were regarded as balanced when $p>0.05$. In the original cohort, cumulative incidence survival curves were obtained with Kaplan-Meier methods.

Considering the selection bias and unmeasured confounders, we additionally used an instrument variate (IVA) to calculate them. We selected the regional utilization rate as the IVA in the two-stage residual inclusion analysis [12-15]. Afterward, another multivariate Cox proportional hazard model, including all covariates and residuals, was presented to illustrate the results more precisely.

Several sensitivity analyses were performed to validate the robustness of the results: (1) analyses of CSM and OM after adjusting propensity scores; (2) inverse probability of treatment weighing (IPTW) and standardized mortality ratio weighting (SMRW) calculated with the propensity score to estimate the relationship between treatment types and outcomes among the entire cohort; (3) analyses of CSM and OM stratified by propensity scores; and (4) adjustment of unbalanced covariates in the matched cohort.

All analyses were performed with the statistical software packages R (http://www.R-project.org, The $\mathrm{R}$ Foundation) and EmpowerStats (http://www. empowerstats.com, X\&Y Solutions, Inc., Boston, MA). A $p$ value of $<0.05$ was considered statistically significant.

\section{Results}

Our study included 19,554 individuals, of who 2,350 received cryotherapy, while 17,204 received RP. 
The inclusion and exclusion criteria are presented in detail in Figure 1. Table 1 shows the patients' baseline characteristics. Patients who received cryotherapy had a higher average age and PSA level than those who received RP. However, the T stage and GS were lower in the cryotherapy group. From the multivariate regression analysis, patients who received cryotherapy had a higher overall risk of death (hazard ratio $[\mathrm{HR}]=4.74,95 \%$ confidence interval $[\mathrm{CI}] 4.20$ 5.35, $p<0.001)$. After adjusting the covariates, including age, PSA, T stage, and GS, cryotherapy still led to a higher overall risk of death than RP (HR = $2.52,95 \%$ CI 1.99-3.20, $p<0.001)$. As for the CSM analyses, results from the crude model indicated that cryotherapy was inferior to RP in decreasing CSM ( $\mathrm{HR}=4.81,95 \%$ CI 3.26-7.09, $p<0.001)$. However, no significant differences were observed after adjusting confounders, with HR $=1.38$ (95\% CI 0.63-3.03, $p=$ $0.41)$.
Patients diagnosed with prostate cancer between 2004-2015 from SEER database

$$
(\mathrm{n}=665054)
$$

Patients with adenocarcinoma of prostate

$$
(\mathrm{n}=638127)
$$

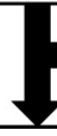

Patients with $\mathrm{PCa}$ as the only tumor

$$
(\mathrm{n}=580 \text { 047) }
$$

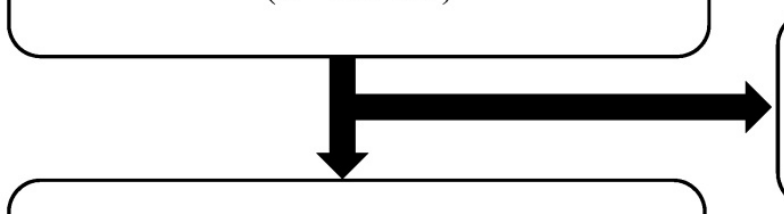

Patients with cT1-T2b N0M0 prostate cancer

$$
(\mathrm{n}=251919)
$$

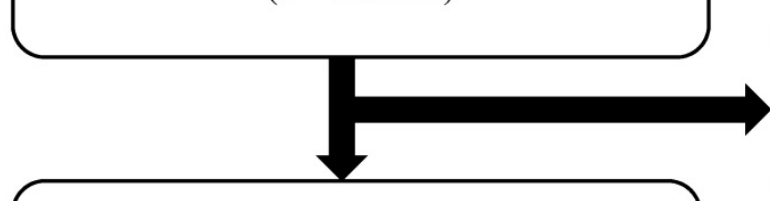

Patients with low risk or intermediate risk

prostate cancer

$(\mathrm{n}=177000)$

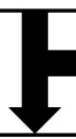

Patients with detailed information on the receipt of RP or cryotherapy

$(\mathrm{n}=19554)$
Histology different from adenocarcinoma or histology unknown

$$
(\mathrm{n}=26 \text { 927) }
$$

Patients 1) not confirmed by positive histology or 2) confirmed by autopsy or 3) accompanied with other kinds of tumor

$$
(\mathrm{n}=58080)
$$

Patients with other stages

$$
(\mathrm{n}=328 \text { 128) }
$$

Patients 1) with GS more than 7 or 2) PSA more than $20 \mathrm{ng} / \mathrm{ml}$

$(\mathrm{n}=74919)$

Patients received other treatment

$$
(\mathrm{n}=157446)
$$

Figure 1. Flowchart describing the selection of patients treated with local treatment or non-local treatment in the Surveillance, Epidemiology and End Results databases, 2004-2015. Abbreviation: RP: radical prostatectomy. 
Table 1. Descriptive characteristics of 19,554 patients that underwent either cryotherapy or radical prostatectomy between 2004 and 2015 from the Surveillance Epidemiology and End Results database

\begin{tabular}{|c|c|c|c|}
\hline & $\mathrm{RP}(\mathrm{N}=17204)$ & Cryotherapy $(\mathrm{N}=2350)$ & $P$ value \\
\hline Age, yr mean \pm SD & $60.32 \pm 7.13$ & $68.91 \pm 7.55$ & $<0.001$ \\
\hline PSA level $(\mathrm{ng} / \mathrm{ml})$, mean \pm SD & $5.85 \pm 3.13$ & $6.72 \pm 3.35$ & $<0.001$ \\
\hline Marital status, n (\%) & & & $<0.001$ \\
\hline Married & $13460(78.24 \%)$ & $1658(70.55 \%)$ & \\
\hline Single & $1596(9.28 \%)$ & $220(9.36 \%)$ & \\
\hline Divorced/Widowed & $1204(7.00 \%)$ & $256(10.89 \%)$ & \\
\hline Unknown & $944(5.49 \%)$ & $216(9.19 \%)$ & \\
\hline Race, $n(\%)$ & & & $<0.001$ \\
\hline White & $14522(84.41 \%)$ & $1857(79.02 \%)$ & \\
\hline Black & $1518(8.82 \%)$ & $370(15.74 \%)$ & \\
\hline Other & $981(5.70 \%)$ & $97(4.13 \%)$ & \\
\hline Unknown & $183(1.06 \%)$ & $26(1.11 \%)$ & \\
\hline T stage, $\mathrm{n}(\%)$ & & & $<0.001$ \\
\hline $\mathrm{T} 1$ & $720(4.19 \%)$ & $1993(84.81 \%)$ & \\
\hline $\mathrm{T} 2 \mathrm{a}$ & $13827(80.37 \%)$ & $242(10.30 \%)$ & \\
\hline $\mathrm{T} 2 \mathrm{~b}$ & $2657(15.44 \%)$ & $115(4.89 \%)$ & \\
\hline Gleason Score, $\mathrm{n}(\%)$ & & & $<0.001$ \\
\hline $3+3$ & $10406(60.49 \%)$ & $1135(48.30 \%)$ & \\
\hline $3+4$ & $5178(30.10 \%)$ & $827(35.19 \%)$ & \\
\hline $4+3$ & $1620(9.42 \%)$ & $388(16.51 \%)$ & \\
\hline $\begin{array}{l}\text { Low risk vs intermediate risk, } \\
\text { n (\%) }\end{array}$ & & & $<0.001$ \\
\hline Low risk & $8388(48.76 \%)$ & $967(41.15 \%)$ & \\
\hline Intermediate risk & $8816(51.24 \%)$ & $1383(58.85 \%)$ & \\
\hline
\end{tabular}

Abbreviations: SD: standard difference; PSA: prostate-specific antigen.

Table 2. Descriptive characteristics of 2,060 patients received RP versus cryotherapy after propensity score matching (ratio 1:1) between 2004 and 2015 from the Surveillance Epidemiology and End Results database

\begin{tabular}{|c|c|c|c|}
\hline & $\mathrm{RP}$ & Cryotherapy & P value \\
\hline Age, yr mean \pm SD & $63.70 \pm 7.78$ & $65.03 \pm 8.06$ & $<0.001$ \\
\hline PSA level $(\mathrm{ng} / \mathrm{ml})$, mean \pm SD & $6.44 \pm 3.43$ & $6.50 \pm 3.73$ & 0.71 \\
\hline Marital status & & & $<0.001$ \\
\hline Married & $787(76.4)$ & $705(68.4)$ & \\
\hline Single & $78(7.6)$ & $116(11.3)$ & \\
\hline Divorced/Widowed & $77(7.5)$ & $119(11.6)$ & \\
\hline Unknown & $88(8.5)$ & $90(8.7)$ & \\
\hline Race & & & $<0.001$ \\
\hline White & $873(84.8)$ & $804(78.1)$ & \\
\hline Black & $87(8.4)$ & $178(17.3)$ & \\
\hline Other & $58(5.6)$ & $36(3.5)$ & \\
\hline Unknown & $12(1.2)$ & $12(1.2)$ & \\
\hline T stage & & & $<0.001$ \\
\hline $\mathrm{T} 1$ & $636(61.7)$ & $673(65.3)$ & \\
\hline $\mathrm{T} 2 \mathrm{a}$ & $340(33)$ & $242(23.5)$ & \\
\hline $\mathrm{T} 2 \mathrm{~b}$ & $54(5.2)$ & $115(11.2)$ & \\
\hline GS biopsy & & & 0.0071 \\
\hline $3+3$ & $578(56.1)$ & $510(49.5)$ & \\
\hline $3+4$ & $311(30.2)$ & $343(33.3)$ & \\
\hline $4+3$ & $141(13.7)$ & $177(17.2)$ & \\
\hline Low risk vs intermediate risk & & & 0.0005 \\
\hline Low risk & $474(46)$ & $395(38.3)$ & \\
\hline Intermediate risk & $556(54)$ & $635(61.7)$ & \\
\hline
\end{tabular}

After propensity score matching, a total of 2,060 patients were screened in the matched cohort, with 1,030 in each treatment group. After matching, the age, $\mathrm{T}$ stage, and GS remained unbalanced (Table 2). In the matched cohort, patients who had undergone cryotherapy had higher OM and CSM $(\mathrm{HR}=2.70$ [95\% CI 1.99-3.66, $p<0.001]$ and HR $=2.99$ [95\% CI $1.19-7.48, p=0.02]$, respectively). After adjusting the imbalanced covariates in the matched cohort, patients who had undergone RP showed a higher survival rate than those who had undergone cryotherapy $(\mathrm{HR}=$ $2.19,95 \%$ CI 1.60-3.00, $p<0.001$ ), while cryotherapy showed non-inferiority in the aspect of CSM (HR = 1.76, 95\% CI 0.68-4.52, $p=0.24)$. In the IV-adjusted analysis (Table 3), cryotherapy was inferior to RP in decreasing $\mathrm{OM}(\mathrm{HR}=2.52,95 \%$ CI 1.99-3.20), while no obvious decrease of CSM was observed in the comparison of RP and cryotherapy ( $\mathrm{HR}=1.38,95 \% \mathrm{CI}$ 0.63-3.03). The subgroup analyses showed that patients with different PSA levels had different results from RP and cryotherapy. Stratified by the PSA level, RP displayed obvious benefits in decreasing CSM (HR $=5.02,95 \%$ CI 1.30-19.39, $p=0.02$ ) (Figure 2). We further used the D'Amico risk classification to divide the patients into low- and intermediate-risk groups. The same benefit of RP was found among those with intermediate-risk PCa (HR $=3.42,95 \%$ CI 2.00-5.84, $p$ $<0.001$ ), while no significant outcome was observed in $\mathrm{GS}=7[(\mathrm{HR}=2.52,95 \% \mathrm{CI} 0.61-10.47, p=0.20)$ for $\mathrm{GS}=3+4$ and $(\mathrm{HR}=1.33,95 \% \mathrm{CI} 0.30-5.83, p=0.70)$ for GS $=4+3$ ], indicating that the PSA level played a more important role than GS.

The sensitivity analyses demonstrated similar survival outcomes in the comparison of cryotherapy and RP. The IPTW- and SMRW-adjusted models both showed no clear superiority in terms of reducing CSM after the adjustment of confounders $(\mathrm{HR}=0.88,95 \%$ CI $0.67-1.15, p=0.35$ and $\mathrm{HR}=0.67,95 \%$ CI 0.41-1.10, $p=0.12$, respectively) (Table S2). The propensity score-adjusted model also reached the same outcome. With regard to a decline in CSM, no obvious differences were observed between the two therapies.

\section{Discussion}

Currently, cryotherapy occurs as a new primary or salvage treatment of PCa since its first use $[16,17]$. Cryotherapy induces tumor apoptosis by promoting protein denaturation and the destruction of cellular membranes by using extremely low temperature, which then leads to vascular stasis and microthrombi and causes ischemic necrosis of the tumor tissue [1821].

Several studies show that focal cryotherapy presents encouraging short-term outcomes [22-25]. However, most of these studies were single-arm designed, with few focusing on the effectiveness comparison of RP and cryotherapy. Our study examined long-term oncologic outcomes with a 
follow-up duration of 72 months (range: 43-103 months). According to our results, for low- and intermediate-risk $\mathrm{PCa}$, cryotherapy shows no significant difference in decreasing CSM compared with RP, but is also no match for RP in OM decline.
A

Age

Q1

Q2

Q3

Q4

T stage

$\mathrm{T} 1$

T2a

$\mathrm{T} 2 \mathrm{~b}$

PSA

$<4$

4-10

$\geq 10$

GS

$3+3$

$3+4$

$4+3$

low vs intermediate

low

intermediate

B

\section{Age}

Q1

Q2

Q3

Q4

T stage

T1

T2a

T2b

PSA

$<4$

4-10

$\approx 10$

GS

$3+3$

$3+4$

$4+3$

low vs intermediate

low

intermediate
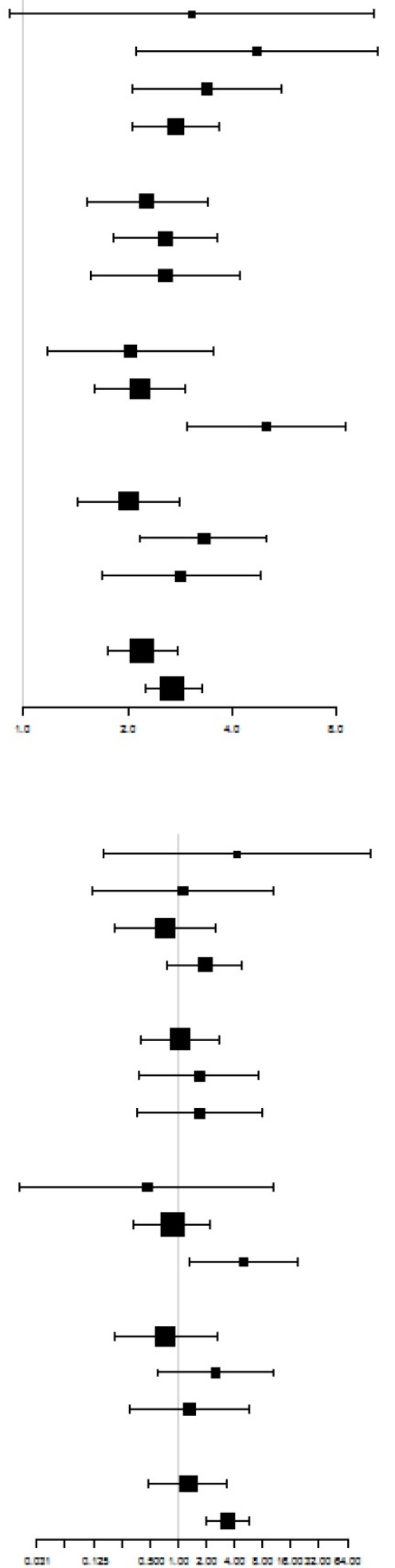

$1.29(0.49,3.39)$

$3.42(2.00,5.84)$
HR

$4.31(0.16,115.30)$

$1.13(0.12,10.39)$

$0.73(0.21,2.57)$

$1.94(0.77,4.88)$

$1.04(0.40,2.71)$

$1.68(0.39,7.28)$

$1.73(0.37,8.08)$

$P$ for interation

0.64

0.47

0.83

0.79

0.25

P for interation

0.94

0.37

0.68

$0.47(0.02,10.48)$

$0.86(0.33,2.22)$

$5.02(1.30,19.39)$

0.42

$0.74(0.21,2.60)$

$2.52(0.61,10.47)$

$1.33(0.30,5.83)$

0.18

Figure 2. Subgroup analyses of OM and CSM in the comparison between RP and cryotherapy. (A) Subgroup analysis of OM in the comparison of RP and cryotherapy. (B) Subgroup analysis of CSM in the comparison of RP and cryotherapy. Abbreviations: OM: Overall mortality; CSM: Cancer specific mortality; RP: Radical prostatectomy; GS: Gleason Score; PSA: Prostate specific antigen; Q1-Q4: Quartile 1 - Quartile 4. 
Table 3. Multivariate cox regression analyses for CSM and OM in the total cohort and matched population

\begin{tabular}{lllll}
\hline Outcome & Treatment & Non-adjusted model & Adjusted model & PSM model \\
\hline CSM & RP & Ref. & Ref. & Ref. \\
& cryotherapy & $4.81(3.26,7.09) \mathrm{p}<0.0001$ & $1.38(0.63,3.03) \mathrm{p}=0.4162$ & $2.99(1.19,7.48) \mathrm{p}=0.0195$ \\
OM & RP & Ref. & Ref. & Ref. \\
& cryotherapy & $4.74(4.20,5.35) \mathrm{p}<0.0001$ & $2.52(1.99,3.20) \mathrm{p}<0.0001$ & $2.70(1.99,3.66) \mathrm{p}<0.0001$ \\
\end{tabular}

Abbreviations: OM: overall mortality; CSM: cancer specific mortality; RP: radical prostatectomy; PSM: propensity score matching, IVA = instrument variable;

Adjusted model: adjusted for age, T stage, Gleason score (GS) and prostate specific antigen (PSA) level;

Propensity score matching (PSM) model: matched according to age, T stage, GS and PSA;

Instrument variate (IVA) adjusted model: adjusted for age, T stage, GS and PSA and residual

A Cryotherapy vs RP $+\mathrm{RP}+$ Cryotherapy

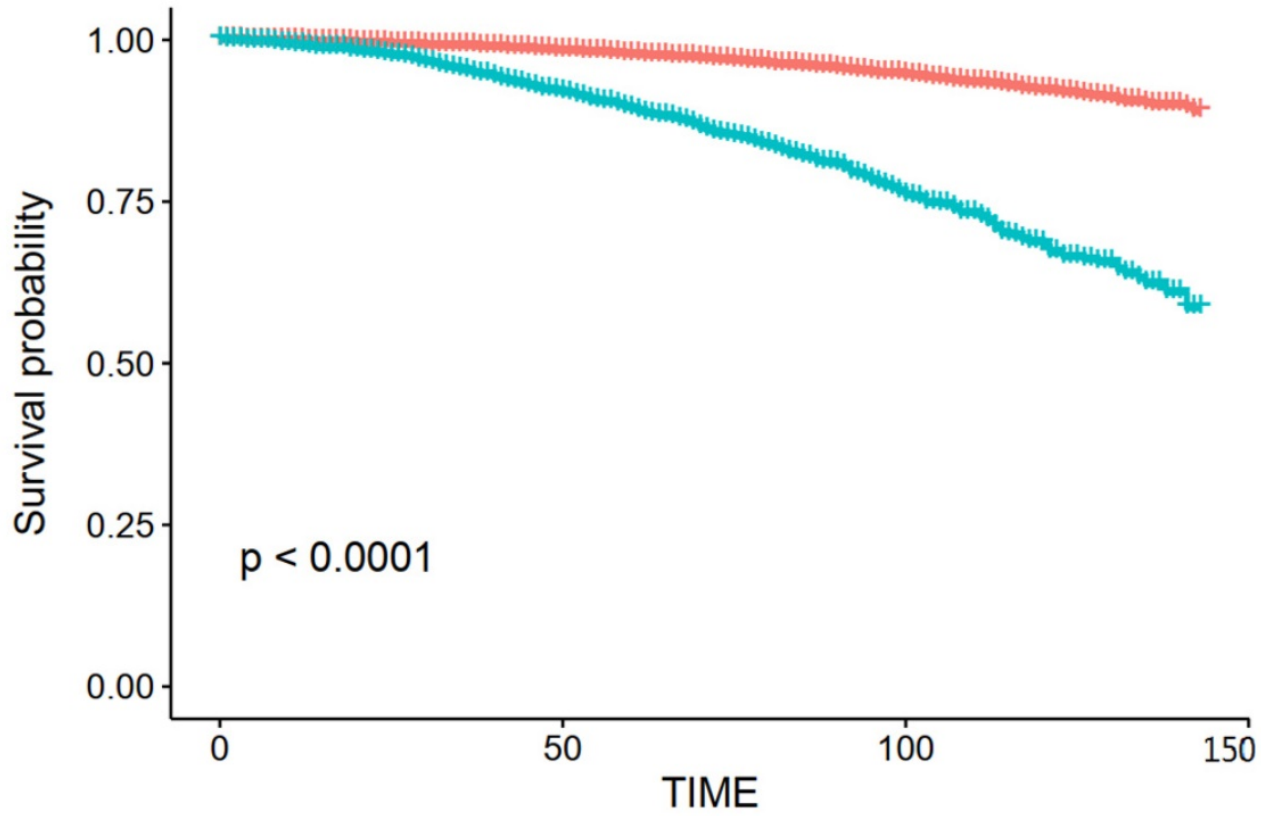

Cryotherapy vs RP $+\mathrm{RP}+$ Cryotherapy

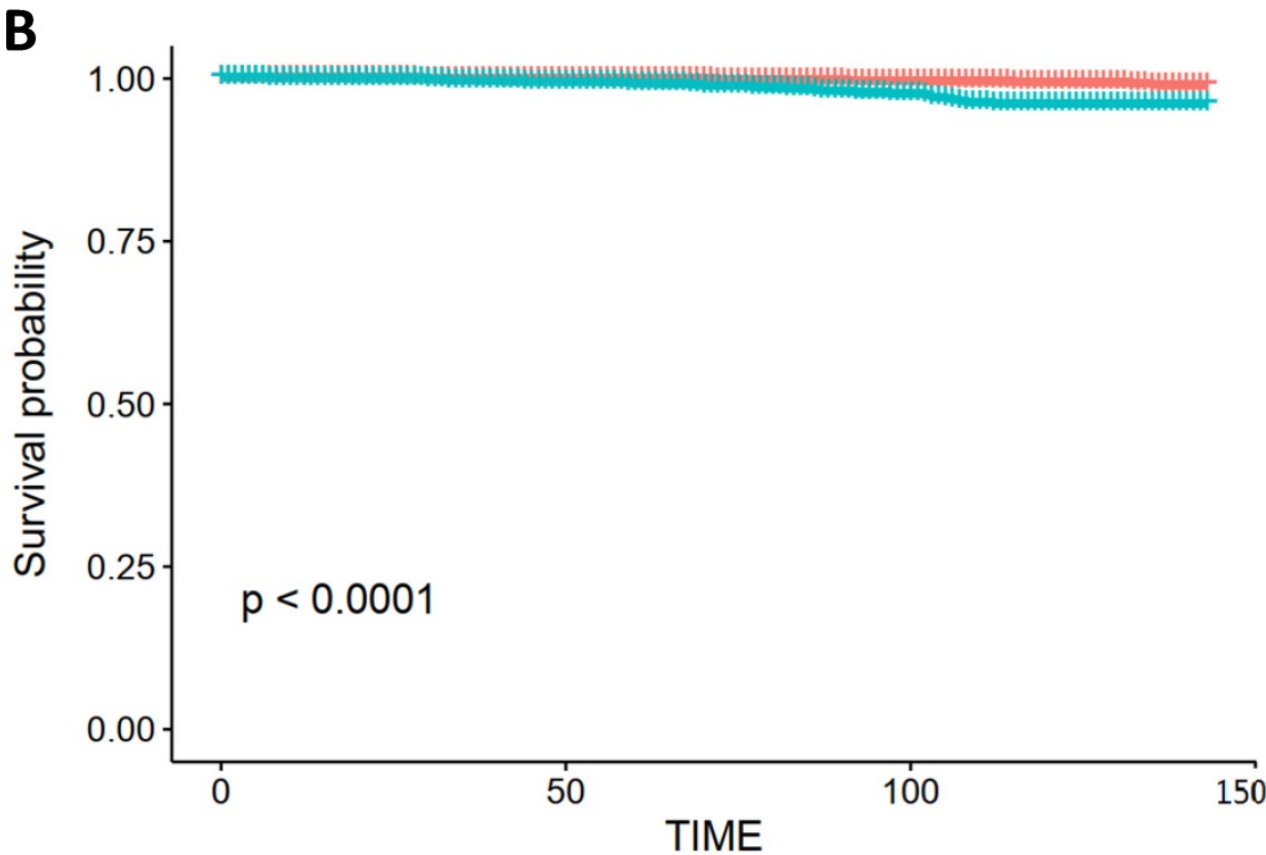

Figure 3. Kaplan-Meier survival curve of OS and CSS. (A) Kaplan-Meier survival curve of OS in the comparison of RP and cryotherapy. (B) Kaplan-Meier survival curve of CSS in the comparison of RP and cryotherapy. Abbreviations: OS: Overall survival; CSS: Cancer specific survival; RP: Radical prostatectomy. 
Additionally, one retrospective matched-pair cohort including 68 individuals in each group showed that oncologic outcomes after focal cryosurgery versus RP were similar in regard to the need for salvage therapy $(p=0.55)$ [26]. This research also reported that those who received cryosurgery had a $70 \%$ reduction in the PSA level (pre-cryotherapy means PSA $5.9 \mathrm{ng} / \mathrm{ml}$ and post-cryotherapy means PSA $1.6 \mathrm{ng} / \mathrm{ml}$ ), indicating that cryosurgery indeed limited the progression of the disease, with an effectiveness similar to RP. In a larger sample size study $(\mathrm{n}=1160)$, patients who received focal cryotherapy showed a $75.7 \%$ biochemical recurrencefree rate at 36 months [27], and the postoperative biopsy was positive in only $3.7 \%$ of patients. Nevertheless, survival outcomes after cryotherapy were not analyzed in this study. Another single-arm study, including 26 participants who underwent focal cryosurgery of the prostate, reported that only three cases experienced biochemical failure, and after salvage conventional definitive therapy, they recovered favorable PSA nadirs [28]. Similar function preservations of cryotherapy were observed in other studies [29-31], but all these failed to compare cryotherapy and RP in the analysis of long-term survival outcomes.

Most research has focused on low- and intermediate-risk PCa, while several studies included patients of high risk. According to Masakatsu Oishi et al. [32], among those who underwent cryotherapy, high-risk patients were more likely to suffer clinical recurrence ( $p=0.046)$, based on the Kaplan-Meier survival curve and trend toward statistical significance $(p=0.06)$ on multivariate analysis. Therefore, the authors suspected that cryotherapy might not be suitable for high-risk PCa, but could become an alternative option for low- and intermediate-risk PCa. Another prospective study that enrolled intermediate- and high-risk individuals showed no discrepancy between the two risk groups [33]. More interestingly, when stratified based on PSA levels at $10 \mathrm{ng} / \mathrm{ml}$, subgroup analyses showed that patients with PSAs higher than $10 \mathrm{ng} / \mathrm{ml}$ were more likely to develop disease recurrence. In this study, the PSA level played a more important role than GS for high-risk PCa, while similar results were observed in our study for those with intermediate-risk PCa. However, more research is needed to explore whether high-risk participants with low PSA levels could benefit from cryotherapy in regard to oncological outcomes, with the exception of functional outcomes.

A major strength of our data comes from the large sample database and direct comparison of cryotherapy and RP. We performed various analyses to verify the effect of cryotherapy on tumor control.
The results all showed that cryotherapy was similar to RP in decreasing CSM. Most studies have focused on the short-term outcomes, such as biochemical recurrence and clinical failure. Our research further explored the survival outcomes and could prove to be a valuable reference in choosing the proper treatment.

Some limitations also need to be highlighted. First, the nature of the retrospective study could not be overcome entirely, although we performed propensity score matching to assume randomization. Second, other assessments of patients were not recorded in the SEER database, such as the Charlson Comorbidity Index and the ECOG score. However, we used an IVA to calculate the unmeasured confounding and make the result more precise. Third, functional outcomes, including urinary incontinence and ejaculation dysfunction (ED), were also not presented in the database. According to the present studies, $0 \%-3.6 \%$ incontinence and $0 \%-42 \%$ ED rates were reported among those who received cryotherapy [34], with fewer complications than RP. In addition, outcomes including disease recurrence and biochemical recurrence were not assessed in our study due to the lack of relevant data. Additionally, as cryotherapy was not implemented in our center, we failed to perform the external validation by using data from our center, thus weaken the accuracy of our results.

\section{Conclusion}

For those with low- and intermediate-risk $\mathrm{PCa}$, cryotherapy could achieve results similar to RP in regard to tumor control. With regard to the preservation of functions, cryotherapy could become a better option. For patients with higher PSA levels, cryotherapy failed to provide survival benefits compared with those who underwent RP. In a word, $\mathrm{RP}$ ranked the best treatment in terms of tumor control, but the advantages of cryotherapy became evident when taking both functional and oncological outcomes into account, especially for low- and intermediate-risk PCa with lower PSA levels.

\section{Supplementary Material}

Supplementary figures and tables. http://www.jcancer.org/v11p5738s1.pdf

\section{Acknowledgements}

All authors contributed to the planning and design of the study. K.J. and S.Q. were involved in review of the raw data and directly involved in the analysis. J.K.L. and X.N.Z. provided analytical feedback based on aggregated results. X.T. and X.Y.L. drafted the manuscript, with input from all authors. Y.Y.L. was responsible for the chart making. All 
authors provided substantive review and commentary on multiple drafts and approved the final version. K.J. and S.Q. had full access to all of the data in the study and takes responsibility for the integrity of the data and the accuracy of the data analysis.

\section{Funding}

This work was supported by the National key research and development program of China (Grant No. 2017YFC0908003, 2017YFC0908004), National Natural Science Foundation of China (Grant No. 81902578, 81974098, 8197032158), China Postdoctoral Science Foundation (2017M612971), Post-doctoral Science Research Foundation of Sichuan University (2020SCU12041), Post-Doctor Research Project, West China Hospital, Sichuan University (2018HXBH084), National Clinical Research Center for Geriatrics, West China Hospital, Sichuan University (Z2018C01).

\section{Competing Interests}

The authors have declared that no competing interest exists.

\section{References}

1. Siegel RL, Miller KD, Jemal A. Cancer statistics, 2017. CA Cancer J Clin. 2017; 67: 7-30.

2. Ferlay J, Soerjomataram I, Dikshit R, et al. Cancer incidence and mortality worldwide: sources, methods and major patterns in GLOBOCAN 2012. Int J Cancer. 2015; 136: E359-86.

3. Heidenreich A, Bellmunt J, Bolla M, et al. EAU guidelines on prostate cancer. Part 1: screening, diagnosis, and treatment of clinically localised disease. Eur Urol. 2011; 59: 61-71.

4. Menon M, Dalela D, Jamil M, et al. Functional recovery, oncologic outcomes and postoperative complications 12 months following robot-assisted radical prostatectomy: follow-up of an evidence-based analysis comparing the Retzius sparing and standard approaches. J Urol. 2018; 199: 1210-7.

5. Hamdy FC, Donovan JL, Lane JA, et al. 10-Year outcomes after monitoring, surgery, or radiotherapy for localized prostate cancer. New Engl J Med. 2016; 375: 1415-24.

6. Ahmed HU, Pendse D, Illing R, et al. Will focal therapy become a standard of care for men with localized prostate cancer? Nat Clin Pract Oncol. 2007; 4: 63242

7. Eggener SE, Scardino PT, Carroll PR, et al. Focal therapy for localized prostate cancer: a critical appraisal of rationale and modalities. J Urol. 2007; 178: 226067.

8. Crawford ED, Al Barqawi MD. Targeted focal therapy: a minimally invasive ablation technique for early prostate cancer. Oncology. 2007; 21: 27-32.

9. Cohen JK, Miller Jr RJ, Ahmed S, et al. Ten-year biochemical disease control for patients with prostate cancer treated with cryosurgery as primary therapy. Urology. 2008; 71:515-8.

10. Ramsay CR, Adewuyi TE, Gray J, et al. Ablative therapy for people with localised prostate cancer: a systematic review and economic evaluation. Health Technol Assess. 2015; 19: 1.

11. Byrd DR, Carducci MA, Compton CC, et al. AJCC cancer staging manual New York: Springer. 2010; 7: 97-100.

12. Robins JM, Hernán MA, Brumback B. Marginal structural models and causal inference in epidemiology. Epidemiology. 2000; 11: 550-560.

13. Cai B, Small DS, Have TRT. Two-stage instrumental variable methods for estimating the causal odds ratio: analysis of bias. Stat Med. 2011; 30: 1809-24.

14. Ying A, Xu R, Murphy J. Two-stage residual inclusion for survival data and competing risks-An instrumental variable approach with application to SEER-Medicare linked data. Stat Med. 2019; 38: 1775-1801.

15. Terza JV. Two-stage residual inclusion estimation in health services research and health economics. Health Serv Res. 2018; 53: 1890-99.

16. Bahn DK, Silverman P, Lee Sr F, et al. Focal prostate cryoablation: initial results show cancer control and potency preservation. J Endourol. 2006; 20: 688-92.

17. Onik G, Vaughan D, Lotenfoe R, et al. The "male lumpectomy": focal therapy for prostate cancer using cryoablation results in 48 patients with at least 2-year follow-up. Urol Oncol. 2008; 26: 500-5.
18. Fahmy WE, Bissada NK. Cryosurgery for prostate cancer. Arch Androl 2003; 49: 397-407.

19. Rees J, Patel B, MacDonagh R, et al. Cryosurgery for prostate cancer. BJU Int. 2004; 93: 710-4.

20. Han KR, Belldegrun AS. Third-generation cryosurgery for primary and recurrent prostate cancer. BJU Int 2004; 93: 14-8.

21. Beerlage HP, Thüroff S, Madersbacher S, et al. Current status of minimally invasive treatment options for localized prostate carcinoma. Eur Urol. 2000; 37: 2-13.

22. Bahn DK, Silverman P, Lee Sr F, et al. Focal prostate cryoablation: initial results show cancer control and potency preservation. J Endourol. 2006; 20: 688-92.

23. Onik G, Vaughan D, Lotenfoe R, et al. The "male lumpectomy": focal therapy for prostate cancer using cryoablation results in 48 patients with at least 2-year follow-up. Urol Oncol. 2008; 26: 500-5.

24. Truesdale MD, Cheetham PJ, Hruby GW, et al. An evaluation of patient selection criteria on predicting progression-free survival after primary focal unilateral nerve-sparing cryoablation for prostate cancer: recommendations for follow up. Cancer J. 2010; 16: 544-9.

25. Ahmed HU, Freeman A, Kirkham A, et al. Focal therapy for localized prostate cancer: a phase I/II trial. J Urol. 2011; 185: 1246-55.

26. Bahn D, de Castro Abreu AL, Gill IS, et al. Focal cryotherapy for clinically unilateral, low-intermediate risk prostate cancer in 73 men with a median follow-up of 3.7 years. Eur Urol. 2012; 62: 55-63.

27. Ward JF, Jones JS. Focal cryotherapy for localized prostate cancer: A report from the national Cryo On-Line Database (COLD) Registry. BJU Int. 2012; 109: $1648-54$.

28. Hale Z, Miyake M, Palacios DA. Focal cryosurgical ablation of the prostate: A single institute's perspective. BMC Urol. 2013; 13: 2.

29. Durand M, Barret E, Galiano M, et al. Focal cryoablation: A treatment option for unilateral low-risk prostate cancer. BJU Int. 2014; 113: 56-64.

30. Lian H, Zhuang J, Yang R, et al. Focal cryoablation for unilateral low intermediate-risk prostate cancer: 63-month mean follow-up results of 41 patients. Int Urol Nephrol. 2016; 48: 85-90.

31. Mendez MH, Passoni NM, Pow-Sang J, et al. Comparison of outcomes between preoperatively potent men treated with focal versus whole gland cryotherapy in a matched population. J Endourol. 2015; 29: 1193-8.

32. Oishi M, Gill IS, Ashrafi AN, et al. Primary whole-gland cryoablation for prostate cancer: biochemical failure and clinical recurrence at 5.6 years of follow-up. Eur Urol. 2019; 75: 208-214.

33. Shah TT, Peters M, Eldred-Evans D, et al. Early-medium-term outcomes of primary focal cryotherapy to treat nonmetastatic clinically significant prostate cancer from a prospective multicentre registry. Eur Urol. 2019; 76: 98-105.

34. Shah TT, Ahmed H, Kanthabalan A, et al. Focal cryotherapy of localized prostate cancer: a systematic review of the literature. Expert Rev Anticancer Ther. 2014; 14: 1337-47. 